\title{
The Effect of Attenuation on Gross Earth Models
}

\author{
R. S. Hart, D. L. ANDerson, AND H. Kanamori \\ Seismological Laboratory, California Institute of Technology, Pasadena, California 91125
}

\begin{abstract}
In most recent free oscillation studies of the earth's interior the effect of absorption upon the eigenperiods of the earth has been ignored. This is equivalent to assuming that the earth is close to perfectly elastic. Since the actual earth is significantly anelastic over seismic frequencies, a frequencydependent correction of the order of $1 \%$ must be applied to the normal mode periods in order that models based on those modes may be compared with body wave results. The eigenperiods of an earth model based on uncorrected data, model C2 (Anderson and Hart, 1976a), are adjusted for attenuation and then inverted to determine the resulting overall perturbation in the earth model. The corrected normal mode data as well as the uncorrected data can be fitted by a spherically symmetric earth model. The effect of including the attenuation term is to generally increase seismic velocities, particularly shear velocities, throughout the model. An important consequence of this change is to reduce or eliminate base line discrepancies between body wave results and normal mode results.
\end{abstract}

\section{INTRODUCTION}

Many attempts have been made in recent years to construct average earth models through the inversion of the observed eigenperiods of the earth [Dziewonski and Gilbert, 1972; Jordan and Anderson, 1974; Gilbert and Dziewonski, 1975; Anderson and Hart, 1976a]. These studies have been successful in matching the observed free oscillation periods to a high degree of precision. In all of these investigations the effect of attenuation on dispersion was assumed to be second order and was ignored despite objections by Jeffreys [1965] and others that the effect was first order based upon the work of Lomnitz [1957], Strick [1967], and Futterman [1962]. Although the eigenperiods have been fit well, serious discrepancies occur between the free oscillation models and body wave data, primarily in the form of large travel time base line shifts. Attempts have been made to attribute these base line shifts to continental-oceanic mantle differences extending to great depth [Jordan, 1975].

Recently, the effect of absorption on dispersion has been reexamined and found, over the seismic band, to be first order [Randall. 1976; Liu et al., 1976; Anderson et al., 1977; Kanamori and Anderson, 1977]. In this paper we consider the effect of correcting the entire spheroidal and toroidal mode set for attenuation on an earth model constructed from uncorrected data, model C2 [Anderson and Hart, 1976a].

In an earlier paper [Hart et al., 1976] we corrected the observed toroidal eigenperiods of the earth for attenuation. These data alone were then used to determine a mantle shear velocity structure appropriate for both $S$ waves and for the observed toroidal modes. In a second paper [Anderson and Hart, $1976 b$ ] we examined the effect of correcting the normal modes for absorption upon the necessity for a shear wave low-velocity zone in the upper mantle: That investigation was in response to earlier criticism by Jeffreys [1965] that such model details based on free oscillations are merely artifacts arising from the neglect of the attenuation effect. We demonstrated that a low-velocity zone was also required by the absorption corrected data. Jeffreys [1965], Carpenter and Davies [1966], and others also suggested that attenuation might be responsible for the base line discrepancies between free oscillations and body waves.

\section{DATA AND INVERSION}

A detailed explanation of the effect of absorption on dispersion is covered by Liu et al. [1976] and Anderson et al.

Copyright (c) 1977 by the American Geophysical Union.

Paper number 6B0923.
[1977]. These authors, and others, have shown that elastic velocities are independent of frequency only at very high and very low frequencies or for a material of infinite $Q$. Seismic frequencies are in a broad absorption band, and observed attenuations are such that seismic velocities do not reflect purely elastic properties nor can they be assumed to be frequency independent. In gross earth modeling it is necessary to correct all the data to a common reference frequency. The resulting elastic properties will then be those which characterize the medium at that particular frequency, with attenuation still present. The elastic properties at any other frequency can then be calculated easily from the reference model. While this procedure will not yield the elastic constants of an equivalent ideally elastic body, it does make all the seismic data consistent. Following Liu et al. [1976] and Anderson et al. [1977], we have assumed a linear superposition model for attenuation in the earth.

We assume that the $Q$ of the medium is frequency independent and that the observed variation of $Q$ among the modes is due entirely to a variation of absorption with depth [Anderson and Archambeau. 1964]. The possibility of an intrinsic frequency dependence of the medium $Q$ over the seismic band cannot be dismissed; present data are not adequate to pursue this possibility further. We have also assumed that all absorption in the mantle is due to losses in shear; this assumption is consistent with the results of Anderson et al. [1965]. Additionally, in a new study of the distribution of attenuation in the earth [Anderson and Hart, 1977] we show that dissipation in pure compression cannot be significant in the mantle. These assumptions permit us to express the attenuation correction in a particularly simple form [Liu et al., 1976]:

$$
\Delta T_{l} / T_{t}=-\left(\pi Q_{t}\right)^{-1} \ln \left(\omega_{R} / \omega_{i}\right)
$$

where $T_{i}$ is the period, $Q_{i}^{-1}$ is the dissipation function, and $\omega_{i}$ is the angular frequency, all referring to the $i$ th mode, and $\omega_{R}$ is the angular reference frequency.

In our investigation we have chosen $\omega_{R}$ to correspond to a period of $1 \mathrm{~s}$. This is approximately the characteristic period of body wave studies and thus allows us to compare directly the present normal mode results to body wave studies. In this case, (1) reduces to

$$
\Delta T_{l} / T_{i}=-\left(\pi Q_{l}\right)^{-1} \ln \left(T_{i}\right)
$$

If the dissipation function $Q^{-1}$ is known for a particular 
TABLE 1. $Q$ Model MM8 [Anderson et al., 1965]

\begin{tabular}{cccc}
\hline $\begin{array}{c}\text { Layer Thickness, } \\
\text { km }\end{array}$ & $\begin{array}{c}\text { Depth to Top } \\
\text { of Layer, } \mathbf{k m}\end{array}$ & $Q_{\beta}$ & $Q_{\boldsymbol{a}}$ \\
\hline 38 & 0 & 450 & 1012 \\
22 & 38 & 60 & 135 \\
10 & 60 & 80 & 180 \\
55 & 70 & 100 & 225 \\
375 & 125 & 150 & 337 \\
100 & 500 & 180 & 405 \\
100 & 600 & 250 & 562 \\
100 & 700 & 450 & 1012 \\
100 & 800 & 500 & 1125 \\
100 & 900 & 600 & 1350 \\
1886 & 1000 & 750 & 1687 \\
3485 & 2886 & $\infty$ & $\infty$ \\
\hline
\end{tabular}

mode, we can readily determine the required correction term. For many modes in the free oscillation data set, $Q$ has been directly measured. Using these observations, we can construct a medium $Q$ versus depth model and compute theoretical $Q$ values for the entire data set. In particular, $Q$ model MM8 [Anderson et al., 1965] fits the observed fundamental toroidal data very closely and is also a good fit to most of the fundamental spheroidal modes. This model is tabulated in Table 1.

Our procedure, then, was to use MM8 to compute the $Q$ and thus obtain the period correction for some 400 theoretical radial, spheroidal, and toroidal eigenperiods computed for model C2 [Anderson and Hart, 1976a]. In Table 2 we have summarized those normal modes which we included in this data set. In this table we have tabulated the fit of model C2 [Anderson and Hart, 1976a] to the observations, the fit of model QM2 to the attenuation corrected observations, and the fit of model QM2 to the corrected C2 theoretical periods. The theoretical $Q$ 's were computed by using the relations given below [Anderson et al., 1965]:

TABLE 2. Normal Mode Data Used in the Inversion

\begin{tabular}{|c|c|c|c|}
\hline \multirow[b]{2}{*}{ Mode Set } & \multicolumn{3}{|c|}{ Average Error, \% } \\
\hline & $\mathrm{C} 2^{*}$ & QM2† & QM2‡ \\
\hline${ }_{0} S_{2-0} S_{29}$ & 0.03 & 0.04 & 0.02 \\
\hline${ }_{0} S_{90-0} S_{98}$ & 0.08 & 0.08 & 0.01 \\
\hline${ }_{1} S_{2}-S_{49}$ & 0.10 & 0.10 & 0.02 \\
\hline${ }_{1} S_{44}-S_{75}$ & 0.07 & 0.07 & 0.02 \\
\hline${ }_{2} S_{3-2} S_{49}$ & 0.07 & 0.07 & 0.02 \\
\hline${ }_{2} S_{57}-{ }_{2} S_{76}$ & 0.15 & 0.16 & 0.02 \\
\hline${ }_{3} S_{1-8} S_{54}$ & 0.05 & 0.04 & 0.02 \\
\hline${ }_{3} S_{58-3} S_{73}$ & 0.11 & 0.10 & 0.01 \\
\hline${ }_{4} S_{2}-{ }_{4} S_{40}$ & 0.08 & 0.08 & 0.01 \\
\hline${ }_{5} S_{2}-{ }_{5} S_{85}$ & 0.09 & 0.08 & 0.01 \\
\hline${ }_{0} S_{0}-S_{8}$ & 0.05 & 0.04 & 0.01 \\
\hline${ }_{0} T_{2-0} T_{29}$ & 0.04 & 0.05 & 0.03 \\
\hline${ }_{0} T_{30-0} T_{48}$ & 0.08 & 0.06 & 0.02 \\
\hline${ }_{1} T_{2-1} T_{29}$ & 0.11 & 0.11 & 0.01 \\
\hline${ }_{1} T_{30-1} T_{80}$ & 0.07 & 0.07 & 0.01 \\
\hline${ }_{2} T_{2-2} T_{81}$ & 0.08 & 0.08 & 0.01 \\
\hline${ }_{9} T_{9-9} T_{72}$ & 0.08 & 0.08 & 0.02 \\
\hline${ }_{4} T_{7}-{ }_{4} T_{60}$ & 0.16 & 0.14 & 0.02 \\
\hline${ }_{5} T_{9-7} T_{48}$ & 0.09 & 0.09 & 0.04 \\
\hline
\end{tabular}

*Relative to uncorrected mode data [Anderson and Hart, 1976a]. † Relative to corrected mode data (data set of Anderson and Hart. [1976a]).

$\ddagger$ Relative to corrected $\mathrm{C} 2$ theoretical periods.

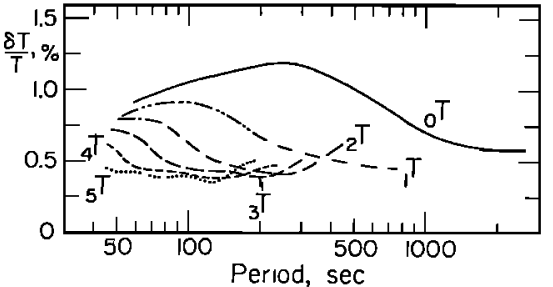

Fig. I. The percentage change in period due to attenuation for the fundamental toroidal modes and for the first five toroidal overtones.

$Q_{T}^{-1}=\sum_{l=1}^{N}\left(\frac{\beta_{l}}{C_{T}} \frac{\partial C_{T}}{\partial \beta_{l}}\right)_{\kappa, \rho, \alpha}{Q_{\beta_{l}}{ }^{-1}}^{-1}$

$$
=\sum_{i=1}^{N}\left(\frac{\partial Q_{T}^{-1}}{\partial Q_{\beta_{l}}^{-1}}\right)_{\kappa} Q_{\beta_{l}}^{-1}
$$

for toroidal oscillations, and

$$
\begin{aligned}
Q_{S}^{-1}= & \sum_{l=1}^{N}\left(\frac{\alpha_{l}}{C_{S}} \frac{\partial C_{S}}{\partial \alpha_{l}}\right)_{\kappa, \rho, \beta} Q_{\alpha_{l}}{ }^{-1}+\left(\frac{\beta_{l}}{C_{S}} \frac{\partial C_{S}}{\partial \beta_{l}}\right)_{\kappa, \rho_{l} \alpha} Q_{\beta_{l}}{ }^{-1} \\
& =\sum_{l=1}^{N}\left[\left(\frac{\partial Q_{S}^{-1}}{\partial Q_{\alpha_{l}}{ }^{-1}}\right) Q_{\alpha_{l}}{ }^{-1}+\left(\frac{\partial Q_{s}^{-1}}{\partial Q_{\beta_{l}}{ }^{-1}}\right) Q_{\beta_{l}}{ }^{-1}\right]_{\kappa}
\end{aligned}
$$

for spheroidal oscillations. The subscript $l$ is the layer index; the subscripts $S, T, \alpha$, and $\beta$ associated with $Q$ identify the wave type. Other subscripts refer to quantities being held constant. $C$ is the phase velocity.

Figure 1 illustrates the percentage change in period for the fundamental and first five overtones of the toroidal modes. Figure 2 gives the percentage change for the corresponding spheroidal eigenperiods. In Figure 2 those modes which correspond to Stoneley waves at the inner core-outer core boundary and at the core-mantle boundary stand out very clearly. These modes have much higher apparent $Q$ 's than adjacent non-Stoneley modes of the same radial overtone number. Hence the percentage period change for such a mode is much smaller, and in Figure 2 these modes show up as deep wells on the period change curves. At the same time the crossover of energy between adjacent radial overtones is clearly illustrated, as these modes alternate as Stoneley modes and ordinary mantle modes.

Model C2 was constructed to include upper- and middlemantle fine structure, determined by high-resolution body wave studies [Helmberger and Wiggins, 1971; Helmberger and Engen, 1974; Hart, 1975] and to satisfy the uncorrected normal mode data. C2 travel times exhibit the same base line shift

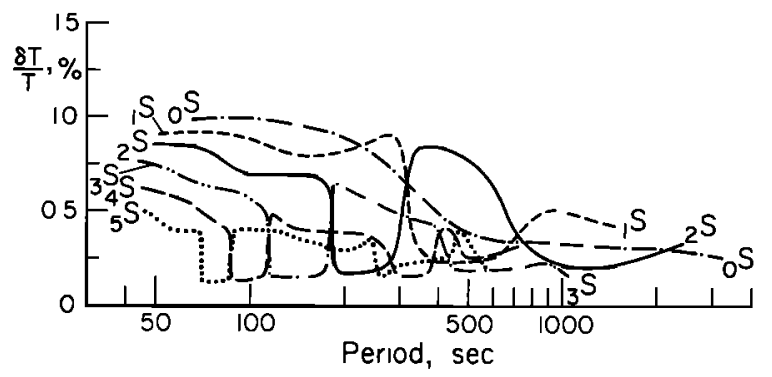

Fig. 2. The percentage change in period due to attenuation for the fundamental spheroidal modes and for the first five spheroidal overtones. 
TABLE 3. Model QM2 and Model C2

\begin{tabular}{|c|c|c|c|c|c|c|}
\hline \multirow[b]{2}{*}{$\begin{array}{c}\text { Radius, } \\
\text { km }\end{array}$} & \multicolumn{3}{|c|}{ Model QM2 } & \multicolumn{3}{|c|}{ Model C2 } \\
\hline & $\begin{array}{c}V_{p} \\
\mathrm{~km} / \mathrm{s}\end{array}$ & $\begin{array}{c}V_{s}, \\
\mathrm{~km} / \mathrm{s}\end{array}$ & $\begin{array}{c}\rho, \\
\mathrm{g} / \mathrm{cm}^{\mathrm{g}}\end{array}$ & $\begin{array}{c}V_{p}, \\
\mathrm{~km} / \mathrm{s}\end{array}$ & $\begin{array}{c}V_{s} \\
\mathrm{~km} / \mathrm{s}\end{array}$ & $\stackrel{\rho,}{\mathrm{g} / \mathrm{cm}^{\mathrm{s}}}$ \\
\hline 6371 & 1.45 & 0.00 & 1.02 & 1.45 & 0.00 & 1.02 \\
\hline 6368 & 1.45 & 0.00 & 1.02 & 1.45 & 0.00 & 1.02 \\
\hline 6368 & 6.50 & 3.72 & 2.80 & 6.50 & 3.72 & 2.80 \\
\hline 6350 & 6.50 & 3.72 & 2.80 & 6.50 & 3.72 & 2.80 \\
\hline 6350 & 8.38 & 4.73 & 3.49 & 8.38 & 4.71 & 3.49 \\
\hline 6330 & 8.38 & 4.73 & 3.50 & 8.38 & 4.73 & 3.51 \\
\hline 6310 & 8.38 & 4.74 & 3.52 & 8.38 & 4.72 & 3.52 \\
\hline 6290 & 8.35 & 4.74 & 3.45 & 8.08 & 4.62 & 3.48 \\
\hline 6270 & 7.82 & 4.58 & 3.39 & 7.93 & 4.36 & 3.44 \\
\hline 6250 & 7.69 & 4.25 & 3.31 & 7.79 & 4.18 & 3.40 \\
\hline 6225 & 7.68 & 4.17 & 3.29 & 7.75 & 4.22 & 3.39 \\
\hline 6200 & 7.74 & 4.27 & 3.31 & 7.78 & 4.30 & 3.37 \\
\hline 6175 & 7.96 & 4.50 & 3.33 & 7.98 & 4.45 & 3.35 \\
\hline 6150 & 8.21 & 4.69 & 3.35 & 8.19 & 4.57 & 3.34 \\
\hline 6125 & 8.45 & 4.76 & 3.36 & 8.40 & 4.62 & 3.34 \\
\hline 6100 & 8.59 & 4.68 & 3.36 & 8.52 & 4.59 & 3.37 \\
\hline 6075 & 8.63 & 4.60 & 3.38 & 8.55 & 4.57 & 3.41 \\
\hline 6050 & 8.65 & 4.57 & 3.43 & 8.58 & 4.57 & 3.47 \\
\hline 6025 & 8.67 & 4.59 & 3.51 & 8.60 & 4.59 & 3.53 \\
\hline 6000 & 8.71 & 4.67 & 3.59 & 8.64 & 4.64 & 3.59 \\
\hline 5983 & 8.85 & 4.77 & 3.63 & 8.79 & 4.71 & 3.62 \\
\hline 5967 & 9.18 & 4.94 & 3.71 & 9.12 & 4.86 & 3.69 \\
\hline 5950 & 9.51 & 5.14 & 3.82 & 9.46 & 5.04 & 3.80 \\
\hline 5925 & 9.54 & 5.14 & 3.81 & 9.50 & 5.07 & 3.79 \\
\hline 5900 & 9.53 & 5.12 & 3.76 & 9.51 & 5.10 & 3.76 \\
\hline 5875 & 9.52 & 5.12 & 3.72 & 9.51 & 5.12 & 3.74 \\
\hline 5850 & 9.70 & 5.29 & 3.73 & 9.70 & 5.26 & 3.76 \\
\hline 5825 & 9.93 & 5.42 & 3.89 & 9.93 & 5.34 & 3.90 \\
\hline 5800 & 10.07 & 5.50 & 3.95 & 10.07 & 5.40 & 3.95 \\
\hline 5775 & 10.06 & 5.50 & 3.97 & 10.08 & 5.42 & 3.98 \\
\hline 5750 & 10.06 & 5.47 & 3.99 & 10.09 & 5.43 & 4.00 \\
\hline 5725 & 10.07 & 5.44 & 4.00 & 10.11 & 5.45 & 4.03 \\
\hline 5700 & 10.21 & 5.58 & 4.04 & 10.25 & 5.60 & 4.07 \\
\hline 5700 & 10.67 & 5.93 & 4.38 & 10.64 & 5.90 & 4.36 \\
\hline 5675 & 10.89 & 6.07 & 4.40 & 10.86 & 6.04 & 4.38 \\
\hline 5660 & 10.94 & 6.09 & 4.42 & 10.91 & 6.06 & 4.40 \\
\hline 5643 & 10.98 & 6.11 & 4.43 & 10.95 & 6.08 & 4.41 \\
\hline 5625 & 10.98 & 6.12 & 4.44 & 10.95 & 6.09 & 4.43 \\
\hline 5602 & 10.99 & 6.16 & 4.47 & 10.96 & 6.13 & 4.46 \\
\hline 5573 & 11.06 & 6.20 & 4.51 & 11.03 & 6.17 & 4.50 \\
\hline 5550 & 11.10 & 6.26 & 4.52 & 11.07 & 6.23 & 4.51 \\
\hline 5500 & 11.20 & 6.33 & 4.55 & 11.17 & 6.31 & 4.54 \\
\hline 5425 & 11.34 & 6.38 & 4.58 & 11.31 & 6.36 & 4.58 \\
\hline 5350 & 11.47 & 6.38 & 4.61 & 11.45 & 6.37 & 4.61 \\
\hline 5275 & 11.60 & 6.40 & 4.64 & 11.58 & 6.38 & 4.64 \\
\hline 5200 & 11.73 & 6.47 & 4.68 & 11.71 & 6.45 & 4.67 \\
\hline 5125 & 11.85 & 6.53 & 4.71 & 11.83 & 6.52 & 4.71 \\
\hline 5050 & 11.97 & 6.58 & 4.74 & 11.95 & 6.56 & 4.74 \\
\hline 4975 & 12.08 & 6.61 & 4.77 & 12.06 & 6.59 & 4.77 \\
\hline 4900 & 12.18 & 6.65 & 4.81 & 12.16 & 6.63 & 4.81 \\
\hline 4825 & 12.27 & 6.69 & 4.85 & 12.26 & 6.67 & 4.84 \\
\hline 4750 & 12.36 & 6.74 & 4.89 & 12.36 & 6.72 & 4.88 \\
\hline 4675 & 12.45 & 6.79 & 4.94 & 12.44 & 6.77 & 4.92 \\
\hline 4600 & 12.54 & 6.83 & 4.97 & 12.54 & 6.81 & 4.96 \\
\hline 4525 & 12.62 & 5.87 & 5.01 & 12.63 & 6.85 & 4.99 \\
\hline 4450 & 12.71 & 6.90 & 5.05 & 12.71 & 6.89 & 5.03 \\
\hline 4375 & 12.80 & 6.93 & 5.09 & 12.80 & 6.92 & 5.07 \\
\hline 4300 & 12.88 & 6.96 & 5.14 & 12.89 & 6.95 & 5.12 \\
\hline 4225 & 12.96 & 6.99 & 5.19 & 12.97 & 6.98 & 5.16 \\
\hline 4150 & 13.04 & 7.02 & 5.24 & 13.04 & 7.01 & 5.22 \\
\hline 4075 & 13.12 & 7.06 & 5.29 & 13.12 & 7.04 & 5.27 \\
\hline 4000 & 13.20 & 7.09 & 5.34 & 13.20 & 7.07 & 5.31 \\
\hline 3925 & 13.28 & 7.13 & 5.38 & 13.28 & 7.11 & 5.36 \\
\hline 3850 & 13.37 & 7.17 & 5.42 & 13.37 & 7.14 & 5.40 \\
\hline 3775 & 13.46 & 7.21 & 5.45 & 13.45 & 7.18 & 5.43 \\
\hline 3700 & 13.54 & 7.24 & 5.48 & 13.53 & 7.21 & 5.45 \\
\hline 3625 & 13.60 & 7.26 & 5.49 & 13.59 & 7.22 & 5.47 \\
\hline 3550 & 13.63 & 7.27 & 5.51 & 13.62 & 7.23 & 5.50 \\
\hline 3510 & 13.64 & 7.27 & 5.52 & 13.63 & 7.24 & 5.50 \\
\hline
\end{tabular}


TABLE 3. (continued)

\begin{tabular}{|c|c|c|c|c|c|c|}
\hline \multirow[b]{2}{*}{$\begin{array}{c}\text { Radius, } \\
\text { km }\end{array}$} & \multicolumn{3}{|c|}{ Model QM2 } & \multicolumn{3}{|c|}{ Model C2 } \\
\hline & $\begin{array}{c}V_{p}, \\
\mathrm{~km} / \mathrm{s}\end{array}$ & $\begin{array}{c}V_{B} \\
\mathrm{~km} / \mathrm{s}\end{array}$ & $\stackrel{\rho,}{\mathrm{g} / \mathrm{cm}^{\mathrm{s}}}$ & $\begin{array}{c}V_{p}, \\
\mathrm{~km} / \mathrm{s}\end{array}$ & $\begin{array}{c}V_{B}, \\
\mathrm{~km} / \mathrm{s}\end{array}$ & $\stackrel{\rho,}{\mathrm{g} / \mathrm{cm}^{\mathrm{s}}}$ \\
\hline 3485 & 13.65 & 7.28 & 5.52 & 13.64 & 7.24 & 5.51 \\
\hline 3485 & 7.98 & 0.00 & 9.97 & 7.98 & 0.00 & 9.96 \\
\hline 3400 & 8.18 & 0.00 & 10.10 & 8.18 & 0.00 & 10.09 \\
\hline 3300 & 8.39 & 0.00 & 10.24 & 8.39 & 0.00 & 10.23 \\
\hline 3200 & 8.55 & 0.00 & 10.38 & 8.55 & 0.00 & 10.37 \\
\hline 3000 & 8.82 & 0.00 & 10.65 & 8.82 & 0.00 & 10.63 \\
\hline 2900 & 8.96 & 0.00 & 10.78 & 8.95 & 0.00 & 10.76 \\
\hline 2800 & 9.08 & 0.00 & 10.91 & 9.07 & 0.00 & 10.89 \\
\hline 2700 & 9.20 & 0.00 & 11.02 & 9.19 & 0.00 & 11.00 \\
\hline 2600 & 9.31 & 0.00 & 11.12 & 9.30 & 0.00 & 11.10 \\
\hline 2500 & 9.42 & 0.00 & 11.21 & 9.41 & 0.00 & 11.20 \\
\hline 2400 & 9.52 & 0.00 & 11.29 & 9.51 & 0.00 & 11.28 \\
\hline 2300 & 9.62 & 0.00 & 11.37 & 9.61 & 0.00 & 11.36 \\
\hline 2200 & 9.72 & 0.00 & 11.45 & 9.71 & 0.00 & 11.44 \\
\hline 2100 & 9.80 & 0.00 & 11.53 & 9.79 & 0.00 & 11.52 \\
\hline 1900 & 9.99 & 0.00 & 11,69 & 9.98 & 0.00 & 11.69 \\
\hline 1800 & 10.07 & 0.00 & 11.78 & 10.06 & 0.00 & 11.77 \\
\hline 1700 & 10.15 & 0.00 & 11.85 & 10.14 & 0.00 & 11.85 \\
\hline 1600 & 10.22 & 0.00 & 11.93 & 10.21 & 0.00 & 11.92 \\
\hline 1500 & 10.25 & 0.00 & 11.99 & 10.24 & 0.00 & 11.99 \\
\hline 1400 & 10.29 & 0.00 & 12.05 & 10.28 & 0.00 & 12.05 \\
\hline 1300 & 10.32 & 0.00 & 12.09 & 10.31 & 0.00 & 12.09 \\
\hline 1215 & 10.34 & 0.00 & 12.12 & 10.33 & 0.00 & 12.12 \\
\hline 1215 & 10.89 & 3.46 & 12.30 & 10.89 & 3.46 & 12.30 \\
\hline 1000 & 11.17 & 3.47 & 12.48 & 11.17 & 3.47 & 12.48 \\
\hline 800 & 11.20 & 3.48 & 12.52 & 11.20 & 3.48 & 12.52 \\
\hline 600 & 11.20 & 3.50 & 12.52 & 11.20 & 3.50 & 12.52 \\
\hline 400 & 11.20 & 3.50 & 12.52 & 11.20 & 3.50 & 12.53 \\
\hline 300 & 11.19 & 3.50 & 12.53 & 11.19 & 3.50 & 12.53 \\
\hline 100 & 11.18 & 3.50 & 12.57 & 11.18 & 3.50 & 12.57 \\
\hline 0 & 11.17 & 3.50 & 12.57 & 11.17 & 3.50 & 12.58 \\
\hline
\end{tabular}

found in other free oscillation studies. The model, however, is very successful in fitting the normal mode data set. It fits the 192 toroidal modes with an average error of $0.09 \%$ and the radial-spheroidal data, 208 modes, with an average error of $0.07 \%$,

The corrected C2 eigenperiods represent our inversion 'data' set, and model $\mathrm{C} 2$ is the starting model. The inversion technique is the linear estimation method described by Jordan and Anderson [1974]. This method employs an iterative algorithm which finds the smallest smooth perturbation to the starting

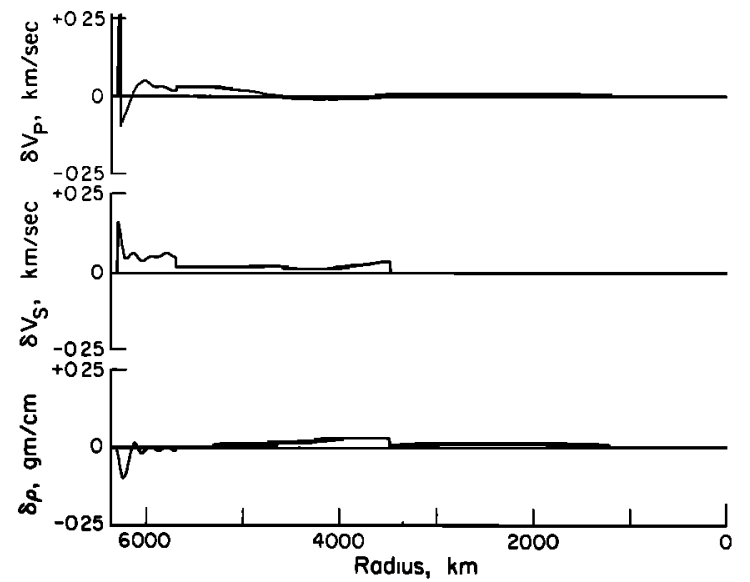

Fig. 3. The perturbation in the model parameters, $V_{p}$ and $V_{s}$, and $\rho$ as a function of radius for the change between model $\mathrm{C} 2$ and model QM2. model that satisfies the inversion data. A detailed description of the theory of the linear inverse problem is given in papers by Backus and Gilbert [1970], Jordan and Franklin [1971], Jordan and Minster [1972], Jordan [1972], and Jordan and Anderson [1974]. The discussions of uniqueness and resolution in the works by Jordan and Anderson [1974] and Anderson and Hart [1976a] apply also to the present paper. The velocities and densities of both model C2 and model QM2 are given in Table 3. Only two iterations were required to produce a model, which we designate QM2, that satisfies the corrected theoretical $\mathrm{C} 2$ periods to within the numerical accuracy of the computation algorithm. This very quick convergence results from the relationship between the $Q$ perturbation kernels and the Frechet velocity kernels used in the inversion (D. L. Anderson et

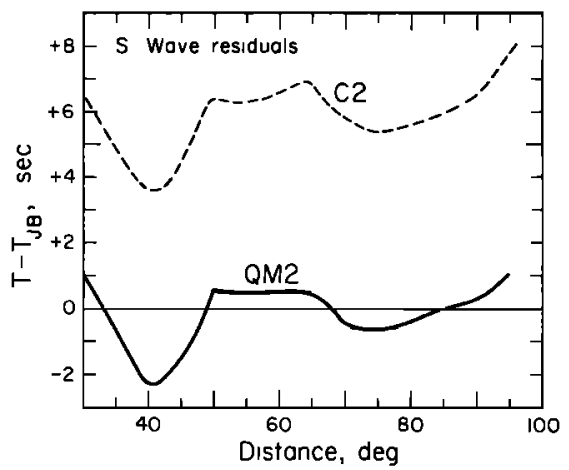

Fig. 4. Residual shear wave travel times relative to the tables of Jeffreys and Bullen [1940] for models C2 and QM2. 
TABLE 4. Surface Focus Shear Wave Travel Times and $d T / d \Delta$

\begin{tabular}{crrrrrr}
\hline & \multicolumn{3}{c}{ Time, s } & & \multicolumn{2}{c}{$d T / d \Delta, \mathrm{s} / \mathrm{deg}$} \\
\cline { 2 - 4 } \cline { 6 - 7 }$\Delta$, deg & \multicolumn{1}{c}{ JB } & HR & QM2 & & HR & QM2 \\
\hline 30 & 670.2 & 669.5 & 671.1 & & 15.4 & 15.5 \\
35 & 748.2 & 749.0 & 747.4 & & 15.3 & 15.1 \\
40 & 824.5 & 825.7 & 822.3 & & 15.2 & 14.9 \\
45 & 897.9 & 899.5 & 896.3 & & 14.5 & 14.7 \\
50 & 968.6 & 970.5 & 969.1 & & 13.9 & 13.9 \\
55 & 1036.8 & 1038.7 & 1037.1 & & 13.4 & 13.4 \\
60 & 1102.6 & 1104.1 & 1103.1 & & 12.8 & 12.9 \\
65 & 1165.5 & 1166.7 & 1166.0 & & 12.2 & 12.1 \\
70 & 1225.6 & 1226.4 & 1255.1 & & 11.7 & 11.6 \\
75 & 1282.6 & 1283.2 & 1282.0 & & 11.1 & 11.1 \\
80 & 1336.5 & 1337.3 & 1336.1 & & 10.5 & 10.6 \\
85 & 1387.3 & 1388.5 & 1387.3 & & 10.0 & 9.8 \\
90 & 1434.5 & 1436.9 & 1434.6 & & 9.4 & 9.1 \\
95 & 1478.2 & 1482.4 & 1479.2 & & 8.8 & 8.8 \\
100 & 1520.4 & & 1522.7 & & 8.4 \\
\hline
\end{tabular}

JB data are from Jeffreys and Bullen [1940]; HR data are from Hales and Roberts [1970].

al., manuscript in preparation, 1977). The fit of model QM2 to the corrected normal mode data is essentially identical to the fit of model $\mathrm{C} 2$ to the uncorrected data.

\section{RESULTS}

In Figure 3 we have plotted the perturbations in the seismic velocities and density as a function of depth for the change from C2 to QM2. The most dramatic effect is an overall increase in shear velocity in the mantle. This increase completely eliminates the large travel time base line shift which characterized model $\mathrm{C} 2$ and other uncorrected earth models. In Figure 4 we have plotted the QM2 and C2 shear travel times as residuals with respect to the tables of Jeffreys and Bullen [1940]. With the exception of the 2-s-deep trough at $40^{\circ}$, required by the shear wave data of Ibrahim and Nuttli [1967] and Hart [1975], the shear wave travel times agree extremely well with those predicted by Jeffreys and Bullen. The theoretical surface-focus shear wave travel times for model QM2 are tabulated in Table 4 . The mean Jeffreys-Bullen residual between $30^{\circ}$ and $95^{\circ}$ is $-0.2 \mathrm{~s}$. In Table 5 we have compiled the deep focus $S c S-S$ differential travel times for models QM2 and C2. The fits of QM2 and $C 2$ are roughly the same, although QM2 does slightly better. We have listed the surface focus $S C S$ travel times for model QM2 and for the Jeffreys-Bullen tables in Table 6. The mean difference over the $30^{\circ}-95^{\circ}$ distance range is less than $-0.2 \mathrm{~s}$.

The surface focus compressional travel times in Table 7 (Table 8 contains the compressional $d T / d \Delta$ ) are slightly slower than those predicted by Herrin's [1968] tables over the $30^{\circ}-95^{\circ}$

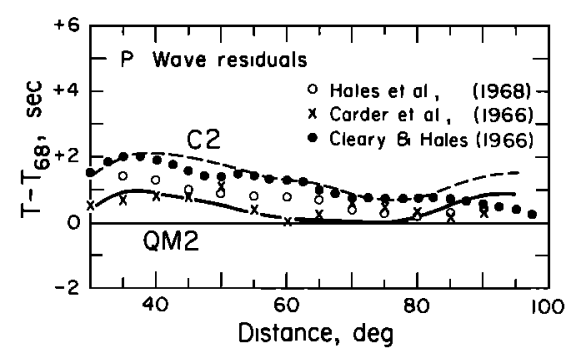

Fig. 5. Residual compressional wave travel times relative to the 1968 tables [Herrin, 1968] for models C2 and QM2. Also shown are the data of Carder et al. [1966], Hales et al. [1968], and Cleary and Hales [1966]. distance range. The mean residual is $+0.5 \mathrm{~s}$. These travel times are plotted as residual times relative to the 1968 tables in Figure 5 along with those of model $\mathrm{C} 2$ and several body wave studies. The QM2 times are about a second faster than the C2 times and fit the observed data of Carder et al. [1966] and Hales et al. [1968] very well. The surface focus $P c P$ travel times for this model are in Table 9. The mean $P c P$ residual, relative to the 1968 tables, is less than $0.1 \mathrm{~s}$. In Table 8 we compare the surface focus $P c P$ - $P$ differential travel times of this model with observed values and with model $\mathrm{C} 2$. The agreement with the observations is about the same for both models.

\section{SUMMARY}

We have examined the effect on a gross earth model, model C2 [Anderson and Hart, 1976a], of correcting the eigenperiods of that model for attenuation. We corrected the periods of 400 radial, spheroidal, and toroidal modes using $Q$ values determined by adopting $Q$ model MM8 [Anderson et al., 1965]. The reference frequency of this correction [see Anderson et al., 1977] was chosen to correspond to a period of $1 \mathrm{~s}$. This is roughly the characteristic period of body waves and allows the results of normal mode studies to be compared directly to those obtained from body waves. Although teleseismic shear waves have dominant periods in the 10 - to 20 -s period range, a period of $1 \mathrm{~s}$ is nevertheless an appropriate value for the characteristic period in the context of travel time studies. Most arrival time readings are performed by picking the first break of the $S$ wave on a seismogram. This portion of the wave form on World-Wide Standard Seismograph Network instruments is dominated by much higher frequencies, probably $0.5 \mathrm{~Hz}$ and less. These normal modes were then inverted to produce a new attenuation corrected earth model, designated QM2.

If $5 \mathrm{~s}$ is taken as the reference period, the change from model $\mathrm{C} 2$ is about $30 \%$ less than in the case of QM2, and the reduction in travel times is also about $30 \%$ less. The residual travel times relative to the Jeffreys-Bullen tables show the same distance dependence as the QM2 residuals but now have a mean residual of $1.0 \mathrm{~s}$ as compared with $-0.2 \mathrm{~s}$ for model QM2.

The new model fits the corrected $\mathrm{C} 2$ eigenperiods to within the numerical accuracy of the computation algorithm and thus fits the observed normal mode data to within $0.08 \%$, as does model C2. We will show in a later paper that for a certain model parameterization the absorption correction to the normal periods can be exactly balanced by a perturbation to the velocity structure. This is what we have demonstrated numerically in the present paper. This new model has higher veloci-

TABLE 5. Deep Focus $S c S-S$ Differential Travel Times in Seconds

\begin{tabular}{ccccc}
\hline$\Delta$, deg & $\begin{array}{c}\text { Observed } \\
\text { Time* }\end{array}$ & $\begin{array}{c}\text { C2 } \\
\text { Time }\end{array}$ & $\begin{array}{c}\text { QM2 } \\
\text { Time }\end{array}$ & $\begin{array}{c}\text { QM2 } \\
\text { Difference }\end{array}$ \\
\hline 30 & $311.3 \pm 1.8$ & 306.8 & 307.0 & -4.3 \\
35 & $259.4 \pm 1.5$ & 258.3 & 258.4 & -1.0 \\
40 & $215.7 \pm 1.6$ & 213.3 & 213.6 & -2.1 \\
45 & $174.3 \pm 1.1$ & 172.2 & 172.3 & -2.0 \\
50 & $138.6 \pm 1.4$ & 137.9 & 137.9 & -0.7 \\
55 & $108.6 \pm 1.4$ & 107.2 & 107.3 & -1.3 \\
60 & $82.0 \pm 1.1$ & 80.7 & 80.8 & -1.2 \\
65 & $59.7 \pm 0.9$ & 59.2 & 59.2 & -0.5 \\
70 & $40.6 \pm 1.0$ & 41.2 & 41.0 & +0.4 \\
75 & $25.5 \pm 1.3$ & 26.6 & 26.1 & +0.6 \\
80 & $14.0 \pm 0.8$ & 15.1 & 14.4 & +0.4 \\
\hline
\end{tabular}

*Jordan and Anderson [1974]; uncertainty is $95 \%$ confidence interval. 
TABLE 6. Surface Focus $P c P$ and $S c S$ Travel Times in Seconds

\begin{tabular}{|c|c|c|c|c|c|c|c|}
\hline \multirow[b]{2}{*}{$\Delta$, deg } & \multicolumn{4}{|c|}{$P_{c P}$} & \multicolumn{3}{|c|}{$S c S$} \\
\hline & JB & $\begin{array}{c}1968 \\
\text { Tables }\end{array}$ & QM2 & $\begin{array}{c}1968 \\
\text { Tables } \\
\text { Difference }\end{array}$ & JB & QM2 & $\underset{\text { Difference }}{\text { JB }}$ \\
\hline 30 & 554.9 & 552.1 & 551.5 & 0.6 & 1011.0 & 1009.4 & 1.6 \\
\hline 35 & 568.6 & 565.9 & 565.3 & 0.6 & 1036.4 & 1034.9 & 1.5 \\
\hline 40 & 583.9 & 581.1 & 580.7 & 0.4 & 1064.6 & 1063.3 & 1.3 \\
\hline 45 & 600.5 & 697.7 & 597.4 & 0.3 & 1095.1 & 1094.2 & 0.9 \\
\hline 50 & 618.3 & 615.5 & 615.2 & 0.3 & 1127.8 & 1127.3 & 0.5 \\
\hline 55 & 637.0 & 634.3 & 634.1 & 0.2 & 1162.5 & 1162.2 & 0.3 \\
\hline 60 & 656.6 & 653.9 & 653.8 & 0.1 & 1198.8 & 1198.8 & 0.0 \\
\hline 65 & 676.9 & 674.2 & 674.2 & 0.0 & 1236.4 & 1236.6 & -0.2 \\
\hline 70 & 697.8 & 695.1 & 695.2 & -0.1 & 1275.2 & 1275.6 & -0.4 \\
\hline 75 & 719.1 & 716.5 & 716.6 & -0.1 & 1315.0 & 1315.5 & -0.5 \\
\hline 80 & 740.6 & 738.0 & 738.3 & -0.3 & 1355.5 & 1356.0 & -0.5 \\
\hline 85 & 762.3 & 759.9 & 760.3 & -0.4 & 1396.5 & 1397.1 & -0.6 \\
\hline 90 & 784.2 & 781.9 & 782.5 & -0.6 & 1437.8 & 1438.5 & -0.7 \\
\hline 95 & & & & & 1479.2 & 1480.2 & -1.0 \\
\hline
\end{tabular}

JB data are from Jeffreys and Bullen [1940]; 1968 tables are from Herrin [1968].

TABLE 7. Surface Focus Compressional Wave Travel Times in Seconds

\begin{tabular}{cccccc}
\hline & & & & & 1968 \\
, deg & JB & HCR & Tables & QM2 & $\begin{array}{c}\text { Tables } \\
\text { Difference }\end{array}$ \\
\hline 30 & 372.5 & 371.0 & 369.5 & 369.9 & 0.4 \\
35 & 416.1 & 414.8 & 413.3 & 414.2 & 0.9 \\
40 & 458.1 & 457.0 & 455.7 & 456.6 & 0.9 \\
45 & 598.9 & 497.4 & 496.4 & 497.2 & 0.8 \\
50 & 538.0 & 536.1 & 535.2 & 535.8 & 0.4 \\
55 & 575.4 & 573.0 & 572.2 & 572.5 & 0.3 \\
60 & 610.7 & 608.2 & 607.4 & 607.5 & 0.1 \\
65 & 644.0 & 641.6 & 640.9 & 641.0 & 0.1 \\
70 & 675.5 & 673.1 & 672.7 & 672.7 & 0.0 \\
75 & 705.0 & 702.9 & 702.6 & 702.6 & 0.0 \\
80 & 732.7 & 730.8 & 730.6 & 730.7 & 0.1 \\
85 & 758.5 & 756.9 & 756.6 & 757.0 & 0.4 \\
90 & 782.7 & 781.1 & 780.7 & 781.3 & 0.6 \\
95 & 805.7 & & 803.9 & 804.5 & 0.7 \\
\hline
\end{tabular}

Average difference is $0.4 \mathrm{~s}$. JB data are from Jeffreys and Bullen [1940]; HCR data are from Hales et al. [1968]; and 1968 tables are from Herrin [1968].

TABLE 8. The $d T / d \Delta$ (in $\mathrm{s} / \mathrm{deg}$ ) for Surface Focus $P$ Waves

\begin{tabular}{cccccc}
\hline$\Delta$, deg & HCR & CGJ & LJ & DJC* & QM2 \\
\hline 30 & 8.94 & 8.88 & 8.92 & $9.13 \pm 0.05$ & 8.96 \\
35 & 8.60 & 8.67 & 8.60 & $8.70 \pm 0.05$ & 8.65 \\
40 & 8.26 & 8.30 & 8.38 & $8.26 \pm 0.07$ & 8.30 \\
45 & 7.91 & 7.99 & 7.90 & $8.11 \pm 0.10$ & 7.92 \\
50 & 7.56 & 7.52 & 7.51 & $7.52 \pm 0.10$ & 7.52 \\
55 & 7.21 & 7.10 & 7.22 & $7.19 \pm 0.08$ & 7.18 \\
60 & 6.86 & 6.84 & 6.75 & $6.95 \pm 0.07$ & 6.85 \\
65 & 6.50 & 6.66 & 6.53 & $6.69 \pm 0.08$ & 6.52 \\
70 & 6.14 & 6.17 & 6.24 & $6.21 \pm 0.09$ & 6.16 \\
75 & 5.77 & 5.77 & 5.83 & $5.88 \pm 0.06$ & 5.80 \\
80 & 5.40 & 5.35 & 5.48 & $5.47 \pm 0.06$ & 5.45 \\
85 & 5.03 & 4.98 & 4.93 & $4.95 \pm 0.06$ & 5.05 \\
90 & 4.66 & 4.74 & 4.65 & $4.60 \pm 0.09$ & 4.74 \\
95 & 4.28 & 4.55 & 4.48 & $4.52 \pm 0.07$ & 4.56 \\
\hline
\end{tabular}

HCR data are from Hales et al. [1968]; CGJ data are from Carder et al. [1966]; LJ data are from Johnson [1967]; and DJC data are from Corbishley [1970].

* Uncertainty is $95 \%$ confidence interval. 
TABLE 9. Surface Focus PcP-P Differential Travel Times in Seconds

\begin{tabular}{|c|c|c|c|c|c|}
\hline$\Delta$, deg & $\mathrm{TJ}$ & $68 \mathrm{M}$ & $\mathrm{C} 2$ & QM2 & $\begin{array}{c}68 \mathrm{M} \\
\text { Difference }\end{array}$ \\
\hline 30 & $181.9 \pm 0.4$ & $181.6 \pm 0.6$ & 181.3 & 181.6 & 0.0 \\
\hline 35 & $151.4 \pm 0.3$ & $151.6 \pm 0.6$ & 150.8 & 151.1 & 0.5 \\
\hline 40 & $125.1 \pm 0.5$ & $124.6 \pm 0.6$ & 123.6 & 124.1 & 0.5 \\
\hline 45 & $100.7 \pm 0.4$ & $100.5 \pm 0.6$ & 99.7 & 100.2 & 0.3 \\
\hline 50 & $79.9 \pm 0.4$ & $79.6 \pm 0.6$ & 79.0 & 79.4 & 0.2 \\
\hline 55 & $62.3 \pm 1.0$ & $61.5 \pm 0.6$ & 61.2 & 61.6 & -0.1 \\
\hline 60 & $46.1 \pm 1.0$ & $45.9 \pm 0.6$ & 45.9 & 46.3 & -0.4 \\
\hline 65 & $33.0 \pm 1.0$ & $32.8 \pm 0.6$ & 32.9 & 33.2 & -0.4 \\
\hline 70 & $22.0 \pm 2.7$ & $22.0 \pm 0.6$ & 22.4 & 22.5 & -0.5 \\
\hline 75 & $13.4 \pm 2.1$ & $13.5 \pm 0.6$ & 14.1 & 14.0 & -0.5 \\
\hline 80 & & $7.2 \pm 0.6$ & 7.7 & 7.6 & -0.4 \\
\hline 85 & & $3.1 \pm 0.6$ & 3.4 & 3.3 & -0.2 \\
\hline 90 & & $1.1 \pm 0.6$ & 1.2 & 1.2 & -0.1 \\
\hline
\end{tabular}

TJ data are from Jordan [1972]; 68M data are from Engdahl and Johnson [1974].

ties, particularly shear velocity, throughout most of the mantle. The largest increases occur in those regions where the $Q$ is the lowest (since the correction to the period is greatest for modes whose sensitivity is large in such regions). This is reflected in the resulting travel times of model QM2. The differential travel times, $P c P-P$ and $S c S-S$, are not changed much from those of model C2. But these data are sensitive only to the lower mantle where $Q$ is higher and consequently, the effect on the eigenperiods is small. Absolute travel times, $P, S$, $P c P$, and $S c S$, are sensitive to the low- $Q$ upper mantle and thus show large changes from $C 2$ to $Q M 2$. Most dramatic is the effect on the travel time of direct $S$ waves. Model C2 and other previous earth models based on normal modes give large, 4- to 8-s, travel time base line shifts relative to the shear wave times of Jeffreys and Bullen [1940]. This discrepancy completely disappears when attenuation is included. Akopyan et al. [1975, 1976] recently obtained a similar result based upon the empirical creep law of Lomnitz [1957]. The resulting agreement in shear wave times is of great importance in our understanding of upper mantle structure and of plate tectonics. There is no longer any need to invoke deep oceanic-continental mantle differences in order to explain the large base line shifts. This study also emphasizes the importance of obtaining accurate measurements of the $Q$ of the earth. The accuracy of gross earth models based on free oscillations in representing the average structure of the earth is directly dependent upon the precision of our knowledge of the $Q$ structure.

Acknowledgments. This research was supported by the Advanced Research Projects Agency of the Department of Defense and was monitored by the Air Force Office of Scientific Research under contract F44620-72-C-0078. We thank Tom Jordan and Richard Sailor for calling our attention to two recent Soviet papers on the subject of this paper. Contribution 2788 of the Division of Geological and Planetary Sciences, California Institute of Technology, Pasadena, California.

\section{REFERENCES}

Akopyan, S. T., V. N. Zharkov, and V. M. Lyubimov, On the dynamic shear modulus of the earth's interior (in Russian), Dokl. Akad. Nauk SSSR, 223, 1975.

Akopyan, S. T., V. N. Zharkov, and V. M. Lyubimov, On the correction of the eigenfrequencies of the earth by the dynamic shear modulus (in Russian), Izv, Akad. Nauk SSR Fiz. Zemli, in press, 1976.

Anderson, D. L., and C. B. Archambeau, The anelasticity of the earth, J. Geophys. Res., 69, 2071, 1964

Anderson, D. L., and R. S. Hart, An earth model based on free oscillations and body waves, J. Geophys Res., 81. 1461, 1976a.

Anderson, D. L., and R. S. Hart, Absorption and the low velocity zone, Nature, 263, 397, $1976 b$.
Anderson, D. L., and R. S. Hart, The $\dot{Q}$ of the earth, submitted to J. Geophys. Res., 1977.

Anderson, D. L., A. Ben-Menahem, and C. B. Archambeau, Attenuation of seismic energy in the upper mantle, J. Geophys. Res., 70, $1441,1965$.

Anderson, D. L., H. Kanamori, R. S. Hart, and H.-P. Liu, The earth as a seismic absorption band, Science, in press, 1977.

Backus, G., and F. Gilbert, Uniqueness in the inversion of inaccurate gross earth data, Phil. Trans, Roy. Soc. London, Ser. A, 266, 123. 1970.

Carder, D. S., D. W. Gordon, and J. N. Jordan, Analysis of surfacefoci travel times, Bull. Seismol. Soc. Amer., 56, 815, 1966.

Carpenter, E. W., and D. Davies, Frequency dependent seismic phase velocities: An attempted reconciliation between the Jeffreys/Bullen and the Gutenberg models of the upper mantle, Nature, 212, 134, 1966.

Cleary, J. R., and A. L. Hales, An analysis of the travel times of $P$ waves to North American stations in the distance range of $32^{\circ}$ to $100^{\circ}$, Bull. Seismol. Soc. Amer., 56, 467, 1966.

Corbishley, D. J., Multiple array measurements of the $P$-wave traveltime derivative, Geophys. J. Roy. Astron. Soc., 19. 1, 1970.

Dziewonski, A., and F. Gilbert, Observations of normal modes from 84 recordings of the Alaskan earthquake of 1964, March 28, Geophys. J. Roy. Astron. Soc., 27, 393, 1972.

Engdahl, E. R., and L. E. Johnson, Differential $P C P$ travel-times and the radius of the core, Geophys. J. Roy. Astron. Soc., 39, 435, 1974.

Futterman, W. I., Dispersive body waves, J. Geophys. Res., 67, 5279, 1962.

Gilbert, F., and A. Dziewonski, An application of normal mode theory to the retrieval of structural parameters and source mechanisms from seismic spectra, Phil. Trans. Roy. Soc. London. Ser. A, $278,187,1975$

Hales, A. L., and J. L. Roberts, Shear velocities in the lower mantle and the radius of the core, Bull. Seismol. Soc. A mer., 60, 1427, 1970.

Hales, A. L., J. R. Cleary, and J. L. Roberts, Velocity distributions in the lower mantle, Bull. Seismol. Soc. Amer., 58, 1975, 1968.

Hart, R. S., Shear velocity in the lower mantle from explosion data, $J$. Geophys. Res., 80, 4889, 1975.

Hart, R. S. D. L. Anderson, and H. Kanamori, Shear velocity and density of an attenuating earth, Earth Planet. Sci. Lett., 32, 25, 1976.

Helmberger, D. V., and G. R. Engen, U pper mantle shear structure, $J$. Geophys. Res., 79, 4017, 1974.

Helmberger, D. V., and R. A. Wiggins, Upper mantle structure of midwestern United States, J. Geophys. Res., 76, 3229, 1971.

Herrin, E. (chairman), 1968 seismological tables for $P$ phases, $B$ ull. Seismol. Soc. Amer., 58, 1193, 1968.

Ibrahim, A. K., and O. Nuttli, Travel-time curves and upper mantle structure from long period $S$ waves, Bull. Seismol. Soc. Amer., 57, $1063,1967$.

Jeffreys, H., The damping of $S$ waves, Nature, 208, 675, 1965.

Jeffreys, H., and K. E. Bullen, Seismological Tables, 55 pp., British Association for the Advancement of Science, London, 1940.

Johnson, L. R., Array measurements of $P$ velocities in the upper mantle, J. Geophys. Res., 72, 6309, 1967.

Jordan, T. H., Estimation of the radial variation of seismic velocities and density in the earth, Ph.D. thesis, Calif. Inst. of Technol., Pasadena, 1972. 
Jordan, T. H., Lateral heterogeneity and mantle dynamics, Nature, 257, 745, 1975.

Jordan, T. H.; and D. L. Anderson, Earth structure from free oscillations and travel-times, Geophys. J. Roy. Astron. Soc., 36, 411 , 1974.

Jordan, T. H., and J. Franklin, Optímal solutions to a linear inverse problem in geophysics, Proc. Nat. Acad. Sci. U.S., 68, 291, 1971.

Jordan, T. H., and J. B. Minster, Applications of a stochastic inverse to the geophysical inverse problem, in The Mathematics of Profile Inversion, edited by L. Colin, Marcell Dekker, New York, 1972.

Kanamori, H., and D. L. Anderson, Importance of physical dispersion in surface wave and free osciliation problems: Review, Rev. Geophys. Space Phys., 15(1), 105, 1977.
Liu, H.-P., D. L. Anderson, and H. Kanamori, Velocity dispersion due to anelasticity: Implications for seismology and mantle composition, Geophys. J. Roy. Astron. Soc., 47, 41, 1976.

Lomnitz, C., Linear dissipation in solids, J. Appl. Phys., 28, 201, 1957. Randall, M. J., Attenuative dispersion and frequency shifts of the earth's free oscillations, Phys. Earth Planet. Interiors, 12, 1, 1976.

Strick, E., The determination of $Q$, dynamic viscosity, and transient creep curves from wave propagation measurements, Geophys. $J$. Roy. Astron. Soc., 13, 197, 1967.

(Received July 23, 1976; revised November 22, 1976; accepted December 12, 1976.) 